\title{
Pulmonary Mycobacterium Avium-Intracellulare Complex Infection in an Infant: A Silent and Coincidental Finding
}

\author{
Vishal Naik, MD, ${ }^{1}$ Pui-Ying Iroh Tam, MD, ${ }^{1, *}$ William Gershan, MD, \\ Andrew A. Colin, MD, and Nadir Demirel, MD ${ }^{1}$
}

An 11-month-old healthy infant girl was noted to have left lower lobe (LLL) opacities on chest X-ray (CXR) after developing desaturations during an elective cochlear implant surgery. Repeat CXR 10 days later revealed hyperexpansion of the left lung and persistent LLL opacity. Chest computerized tomography revealed enlarged mediastinal lymph nodes, left mainstem bronchial obstruction, and nodular opacities. Bronchoscopic biopsy of the endobronchial tissue revealed multiple necrotizing granulomas and grew Mycobacterium avium-intracellulare, Streptococcus viridans, and Actinomyces odontolyticus. This case illustrates the potential for significant mediastinal lymphadenopathy, and endobronchial and parenchymal lesions caused by nontuberculous mycobacteria, which can present insidiously and without respiratory symptoms in otherwise healthy infants, despite advanced pulmonary disease.

Keywords: nontuberculous, mycobacteria, pediatric, endobronchial, mycobacterium avium

\section{Introduction}

$\mathrm{T}$ HE CLASSIFICATION OF nontuberculous mycobacteria (NTM) applies to species of bacteria sharing the same genus as Mycobacterium tuberculosis. The most common NTMrelated disease in the United States is caused by Mycobacterium avium-intracellulare complex (MAC) species. ${ }^{1}$ Ubiquitous in the environment, NTM are primarily a concern for children with chronic pulmonary disease or immunodeficiencies. ${ }^{2}$ Recently, invasive mediastinal and endobronchial MAC infections have been described in immunocompetent children. ${ }^{3-5}$ We describe an insidious presentation of a pulmonary MAC infection in an otherwise asymptomatic infant. Informed consent was obtained from patient's parents to publish the case information and accompanying images and laboratory studies.

\section{Case Report}

An 11-month-old girl with hearing loss secondary to congenital cytomegalovirus (CMV) infection presented for cochlear implant surgery. She was born full term without complications. Her CMV was treated successfully with valganciclovir for 6 months. The patient had no history of any prior respiratory morbidity during her lifetime. Before cochlear im- plant surgery, decreased left-sided breath sounds were noted by the anesthesiologist and the infant developed intermittent desaturations during the procedure (lowest $\mathrm{SpO}_{2} 85 \%$ ). A chest X-ray (CXR) showed a round opacity in the left lower lobe (LLL). The patient tolerated the procedure well and was discharged the next day. Repeat CXR 10 days later showed hyperexpansion of the left lung and a persistent LLL opacity (Fig. 1a). Chest computed tomography (CT) revealed a subcarinal mass with central necrosis significantly obstructing the left mainstem bronchus, hilar lymphadenopathy, hyperexpansion of the left lung, and nodular opacities of the LLL (Fig. 1b-d).

A flexible bronchoscopy revealed an endobronchial polypoid lesion obstructing most of the left mainstem bronchus (Fig. 2a). Bronchoalveolar lavage (BAL) revealed 23\% lymphocytes, $77 \%$ macrophages, and positive PCR analysis for CMV and adenovirus. In the context of a large endobronchial lesion, these viruses were considered incidental findings. Due to high suspicion for $M$. tuberculosis infection, the patient was started on conventional antituberculous therapy (isoniazid, rifampin, ethambutol, and pyrazinamide). Gastric aspirate cultures, acid fast bacillus (AFB) stains, tuberculosis skin test (TST), and Interferon-Gamma Release Assay were all negative. Serologies and urine antigens for fungal etiologies were also negative.

\footnotetext{
${ }^{1}$ University of Minnesota, Minneapolis, Minnesota.

${ }^{2}$ University of Miami, Miami, Florida.

*Current affiliation: Malawi-Liverpool Wellcome Trust, Blantyre, Malawi.
}

(C) Vishal Naik et al. 2017; Published by Mary Ann Liebert, Inc. This article is available under the Creative Commons License CC-BY-NC (http://creativecommons.org/licenses/by-nc/4.0). This license permits non-commercial use, distribution and reproduction in any medium, provided the original work is properly cited. Permission only needs to the obtained for commercial use and can be done via RightsLink. 

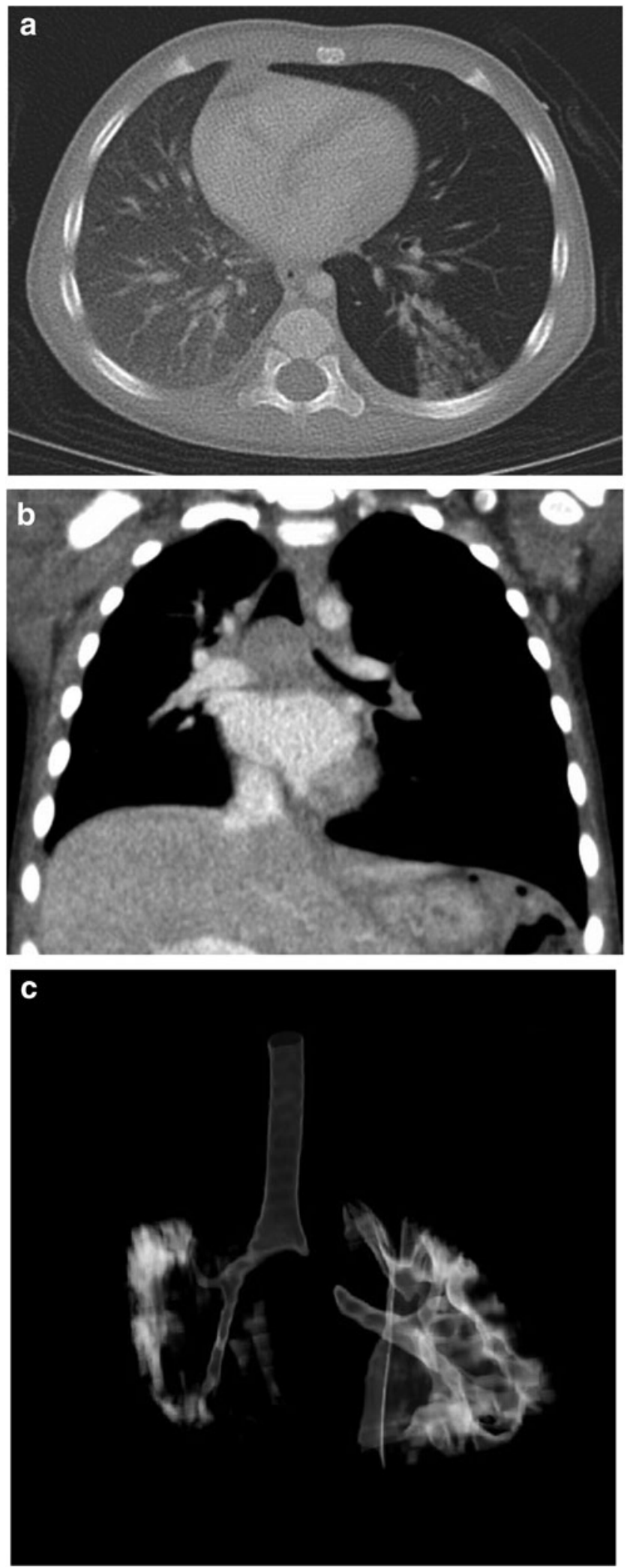

FIG. 1. (a) Chest CT coronal view showing hyperexpansion of the left lung with right shift of mediastinum, a subcarinal mass with central necrosis, near complete obstruction of the left mainstem bronchus. (b) Chest CT axial view showing hyperexpansion of the left lung with right shift of mediastinum and left lower lobe opacities. (c) Chest CT coronal view threedimensional reconstruction showing level of obstruction of the left mainstem bronchus. CT, computed tomography.
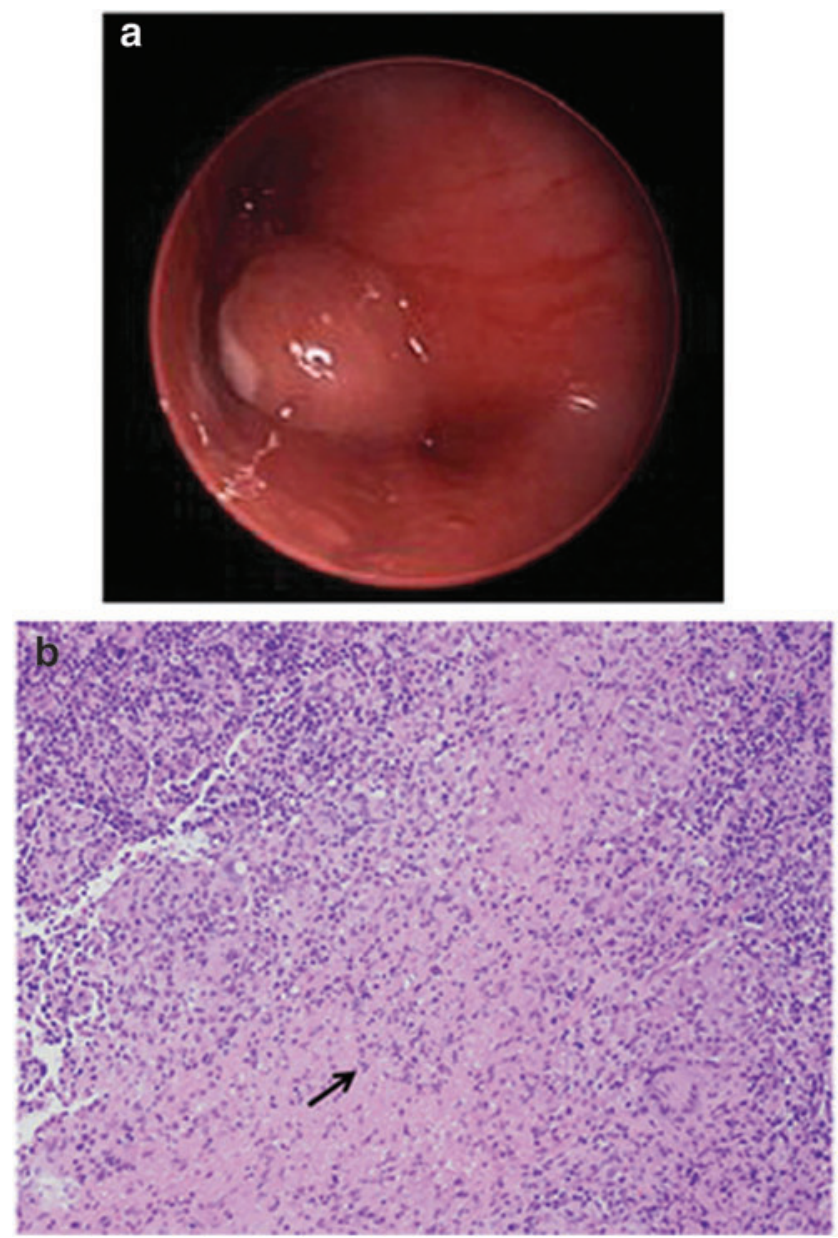

FIG. 2. (a) Picture of bronchoscopy showing mass obstructing $>90 \%$ of the left mainstem bronchus. (b) Picture of biopsy of the polypoid endobronchial lesion showing granulomas with necrosis (arrow). Color images available online at www.liebertpub.com/ped

A rigid bronchoscopy with excisional biopsy of the polypoid endobronchial lesion revealed multiple granulomas with necrosis on pathologic examination (Fig. 2b). Stains for CMV, fungal elements, and AFB were negative. BAL and tissue cultures grew MAC, Actinomyces odontolyticus, and Streptococcus viridans with gastric aspirate cultures remaining negative.

The patient's antibiotic regimen was modified to rifampin, ethambutol, and azithromycin. A 4-month course of clindamycin was added to cover A. odontolyticus and S. viridans.

An extensive immune workup was performed including complete blood count with differential, $\mathrm{T}$ and $\mathrm{B}$ cell subsets, lymphocyte mitogen proliferation, immunoglobulins and IgG subclasses, vaccine titers against Haemophilus influenzae type b, pneumococcus, diphtheria, and tetanus, and interferon gamma RB1 mutations, which were all normal. Next-generation sequencing was performed to evaluate for primary immunodeficiency revealing no clinically relevant genetic variants in 9 genes (CYBB, GATA2, IFNGR1, IFNGR2, IL12B, IL12RB1, IRF8, ISG15, and STAT1). However, a variant was found in the IFNGR1 gene encoding the IFNg R1 protein cited to be a benign polymorphism with no effect on function. Antibody testing for human immunodeficiency virus was negative and as noted above, the patient's cellular immunity was intact. Further HIV test with PCR was not performed at this time. An evaluation for 
cystic fibrosis (CF; newborn screen was negative) and primary ciliary dyskinesia was not performed given absence of typical clinical findings. Pulmonary actinomycosis may result from occult aspiration; however, given absence of clinical signs and risk factors for aspiration (absence of laryngeal cleft and vocal cord paralysis, normal neurological exam, normal brain magnetic resonance imaging, and absence of lipid laden macrophages), a formal evaluation of swallow function was not performed.

Two months after starting treatment, repeat bronchoscopy was performed revealing decreased left mainstem bronchial obstruction; repeat BAL cultures were negative. The antimycobacterial regimen was continued for a total of 12 months. A repeat chest CT at that time showed complete resolution of subcarinal and hilar lymphadenopathy and parenchymal opacities. The patient continued to gain weight appropriately (weight percentile remained between $25 \%$ and $30 \%$ ).

\section{Discussion}

NTM are found primarily in the soil and water sources and transmitted by direct inoculation and aquatic transmission. Although generally not thought to be the cause of invasive disease in immunocompetent children, NTM are most commonly associated with cervical lymphadenitis. ${ }^{6}$ NTM are emerging in individuals with preexisting lung disease such as $\mathrm{CF}^{2}$. Mild forms of primary ciliary dyskinesia and $\mathrm{CF}$ can be underlying conditions for MAC infection. Even though this would be unusual, it should be kept in mind during long-term follow-up.

The diagnosis of pulmonary MAC disease in pediatric patients is difficult with signs and symptoms being nonspecific (chronic cough, sputum production, dyspnea, stridor, fever, or hemoptysis). ${ }^{5}$ Nolt et al. reported 43 children with intrathoracic NTM disease who fell into 2 clinical categories: (1) Patients with respiratory symptoms alone, or (2) Patients with respiratory symptoms and signs of systemic illness including weight loss, anorexia, and fever. ${ }^{3}$ The majority of reported cases presented with cough and wheezing and half of the patients had associated fever. ${ }^{3,7}$ Asymptomatic presentations of intrathoracic MAC in pediatric patients, however, are uncommon. Our case is remarkable for the silent clinical picture despite extensive lung disease and significant obstruction of a main bronchus by a large intrabronchial mass. Only one other case of an asymptomatic infant with NTM has been described, involving an infant diagnosed based on routine TST positivity at the 12-month well child visit. ${ }^{4}$

Pulmonary NTM disease in children is distinct from the isolated pulmonary NTM disease reported in adults. Chest CT in these older individuals typically shows parenchymal disease with nodular infiltrates and bronchiectasis. As opposed to the disease seen in immunocompetent adults, intrathoracic NTM disease in children appears to spare lung parenchyma, and immunologic abnormalities have not been identified. ${ }^{4}$ Pediatric cases of intrathoracic MAC typically present with subcarinal and hilar lymphadenitis and/or endobronchial lesions. ${ }^{3-5}$ Based on chest CT findings, clinical presentation, and response to therapy, we speculate that our patient is the first described pediatric non-CF MAC case presenting with radiologic evidence of parenchymal lung disease (Fig. 1b).

Apart from the ATS recommendations (mostly addressing adult NTM disease) and new guidelines for NTM in individuals with $\mathrm{CF}$, no specific guidelines exist for the diagnosis and treatment of pulmonary NTM in immunocompetent non-CF children. ${ }^{1,2}$ Favorable results with surgical treatment have been reported in immunocompetent children with intrathoracic MAC. ${ }^{7}$ However, as presented in our patient and others, combination antimycobacterial therapy seems to be prudent and effective. ${ }^{4}$

From this report, we suggest that pulmonary NTM disease in otherwise healthy children may present insidiously without respiratory symptoms despite significant lung disease. Pulmonary MAC in immunocompetent children can present with mediastinal lymphadenitis, endobronchial lesions, and parenchymal infiltration. In such cases, awareness of NTM's possibility coupled with a high degree of clinical suspicion may lead to prompt diagnosis and favorable results with antimycobacterial therapy.

\section{Consent}

Informed consent was obtained from patient's parents to publish the case information and accompanying images and laboratory studies.

\section{Author Disclosure Statement}

The authors have indicated they have no financial relationships relevant to this article to disclose.

\section{References}

1. Griffith DE, Aksamit T, Brown-Elliott BA, Catanzaro A, Daley C, Gordin F, et al. An official ATS/IDSA statement: diagnosis, treatment, and prevention of nontuberculous mycobacterial diseases. Am J Respir Crit Care Med 2007; 175:367-416.

2. Floto RA, Olivier KN, Saiman L, Daley CL, Herrmann JL, Nick JA, et al. US Cystic Fibrosis Foundation and European Cystic Fibrosis Society consensus recommendations for the management of non-tuberculous mycobacteria in individuals with cystic fibrosis: executive summary. Thorax 2016; 71:88-90.

3. Nolt D, Michaels MG, Wald ER. Intrathoracic disease from nontuberculous mycobacteria in children: two cases and a review of the literature. Pediatrics 2003; 112:e434.

4. Freeman AF, Olivier KN, Rubio TT, Bartlett G, Ochi JW, Claypool RJ, et al. Intrathoracic nontuberculous mycobacterial infections in otherwise healthy children. Pediatr Pulmonol 2009; 44:1051-1056.

5. del Rio Camacho G, Soriano Guillen L, Flandes Aldeyturriaga J, Hernandez Garcia B, Bernacer Borja M. Endobronchial atypical mycobacteria in an immunocompetent child. Pediatr Pulmonol 2010; 45:511-513.

6. Wolinsky E. Mycobacterial lymphadenitis in children: a prospective study of 105 nontuberculous cases with longterm follow-up. Clin Infect Dis 1995; 20:954-963.

7. Kroner C, Griese M, Kappler M, Schoen C, Hoffmann F, Nicolai $\mathrm{T}$, et al. Endobronchial lesions caused by nontuberculous mycobacteria in apparently healthy pediatric patients. Pediatr Infect Dis J 2015; 34:532-535.

Address correspondence to: Nadir Demirel, $M D$

Division of Pediatric Pulmonology Masonic Children's Hospital University of Minnesota 420 Delaware Street SE, MMC 742 Minneapolis, MN 55455

E-mail: ndemirel@umn.edu

Received for publication April 11, 2017; accepted after revision October 23, 2017. 